\title{
On-Site Genomic Epidemiological Analysis of Antimicrobial-Resistant Bacteria in Cambodia With Portable Laboratory Equipment
}

\begin{abstract}
Aki Hirabayashi', Hideji Yanagisawa ${ }^{2}$, Hiromizu Takahashi ${ }^{3}$, Koji Yahara', Philipp Boeing ${ }^{4}$, Bethan Wolfenden ${ }^{4}$, Vandarith Nov ${ }^{5}$, Vichet Lorn ${ }^{5}$, Mom Veng, Vuth Ann ${ }^{5}$, Chau Darapheak ${ }^{5}$, Keigo Shibayama ${ }^{6 *}$ and Masato Suzuki ${ }^{1 *}$
\end{abstract}

OPEN ACCESS

Edited by:

Jian-Hua Liu,

South China Agricultural University,

China

Reviewed by: Andres Felipe Opazo-Capurro, University of Concepcion, Chile Anusak Kerdsin,

Kasetsart University, Thailand

${ }^{*}$ Correspondence: Keigo Shibayama keigo@nih.go.jp Masato Suzuki suzuki-m@nih.go.jp

Specialty section:

This article was submitted to Antimicrobials, Resistance and Chemotherapy,

a section of the journal Frontiers in Microbiology

Received: 03 March 2021 Accepted: 22 April 2021

Published: 13 May 2021

Citation:

Hirabayashi A, Yanagisawa $H$, Takahashi $H$, Yahara K, Boeing $P$, Wolfenden B, Nov $V$, Lorn $V$, Veng $M$, Ann V, Darapheak C, Shibayama K and Suzuki M (2021) On-Site Genomic Epidemiological Analysis of Antimicrobial-Resistant Bacteria in Cambodia With Portable Laboratory Equipment.

Front. Microbiol. 12:675463. doi: $10.3389 /$ fmich.2021.675463

\begin{abstract}
${ }^{1}$ AMR Research Center, National Institute of Infectious Diseases, Tokyo, Japan, ${ }^{2}$ MicroSKY Lab., Inc., Tokyo, Japan, ${ }^{3}$ Department of General Medicine, Juntendo University School of Medicine, Tokyo, Japan, ${ }^{4}$ Bento Bioworks Ltd., London, United Kingdom, ${ }^{5}$ National Institute of Public Health, Phnom Penh, Cambodia, ${ }^{6}$ Department of Bacteriology II, National Institute of Infectious Diseases, Tokyo, Japan
\end{abstract}

The rapid emergence of carbapenemase-producing gram-negative bacteria (CPGNB) is a global threat due to the high mortality of infection and limited treatment options. Although there have been many reports of CPGNB isolated from Southeast Asian countries, to date there has been no genetic analysis of CPGNB isolated from Cambodia. Sequence-based molecular epidemiological analysis enables a better understanding of the genotypic characteristics and epidemiological significance of antimicrobial-resistant (AMR) bacteria in each country, and allows countries to enact measures related to AMR issues. In this study, we performed on-site genomic epidemiological analysis of CPGNB isolated in Cambodia using a portable laboratory equipment called Bento Lab, which combines a PCR thermal cycler, microcentrifuge, gel electrophoresis apparatus, and LED transilluminator, along with the MinION nanopore sequencer. PCR targeting of major carbapenemase genes using Bento Lab revealed that two Escherichia coli isolates and one Acinetobacter baumannii isolate harbored carbapenemase genes: bla NDM $_{1}$ bla OXA $-48_{3}$, and blaOXA-23, respectively. The results of phenotypic diagnostic tests for CPGNB, such as the carbapenem inactivation method and double-disk diffusion test using a specific inhibitor of metallo- $\beta$-lactamases, were consistent with their AMR genotypes. Whole-genome sequencing analysis using MinION revealed that bla $\mathrm{NDM}_{-5}$ gene was carried on a 93.9-kb plasmid with IncFIA/IncFIB/IncFII/IncQ1 replicons, and blaOXA-181 gene was carried on a 51.5$\mathrm{kb}$ plasmid with the IncX3 replicon in $E$. coli isolates. blaOXA-23 was encoded in two locations on the chromosome of $A$. baumannii. Plasmids carrying bla $a_{\mathrm{NDM}-5}$ or blaOXA-181 in E. coli were highly structurally identical to plasmids prevalent in Enterobacterales in China and other countries, suggesting that they disseminated from a common evolutionary origin. Our findings demonstrate the potential impact of portable laboratory equipment on AMR bacteria research in hospitals and research centers with limited research facilities, and provide the first glimpse into the genomic epidemiology of CPGNB in Cambodia.

Keywords: AMR, carbapenemase, CPE, Acinetobacter, Cambodia 


\section{INTRODUCTION}

Antimicrobial-resistant (AMR) bacteria have emerged and spread all over the world. Among antimicrobials, carbapenems are one of the most reliable last-resort antimicrobials for infections caused by AMR gram-negative bacteria. One of the mechanisms of AMR is drug inactivation mediated by acquired enzymes, such as $\beta$-lactamases (Santajit and Indrawattana, 2016). Among $\beta$-lactamases, extended-spectrum $\beta$-lactamases (ESBLs) and carbapenem-hydrolyzing $\beta$-lactamase (carbapenemases) are clinically important, as ESBLs hydrolyze a broad range of $\beta$-lactams, including cephalosporins, and carbapenemases hydrolyze most $\beta$-lactams, including carbapenems. $\beta$-lactamases are classified using the Ambler scheme as follows. Ambler class A includes ESBLs, such as CTX-M, as well as carbapenemases, such as KPC; class B includes metallo- $\beta$-lactamases (MBLs), such as NDM, IMP, and VIM; class $\mathrm{C}$ includes AmpC $\beta$-lactamases; and class $\mathrm{D}$ includes carbapenem-hydrolyzing oxacillinases, of which OXA-48 is prevalent in Enterobacterales, and OXA-23, OXA-24, and OXA-58 are prevalent in Acinetobacter spp. ESBL and carbapenemase genes are predominantly encoded on conjugative plasmids and have been transferred among Enterobacterales and other gram-negative bacteria (Tzouvelekis et al., 2012). Moreover, carbapenemase-producing gram-negative bacteria (CPGNB) harboring carbapenemase genes often co-harbor clinically relevant antimicrobial resistance genes, such as aminoglycoside and fluoroquinolone resistance genes (Wu et al., 2007; Chmelnitsky et al., 2008; Poirel et al., 2011a). There is great concern about the global spread of plasmids that carry multiple AMR genes that can be transferred between homogeneous and heterogeneous species.

Although CPGNB has been detected in large numbers in Southeast Asia, the publicly available information on CPGNB is limited to a small number of countries (Malchione et al., 2019). In 2015, the World Health Organization (WHO) adopted a global action plan on AMR and launched the Global Antimicrobial Resistance Surveillance System (GLASS), the first global collaborative report to standardize AMR surveillance (WHO, 2015; Tornimbene et al., 2018). Cambodia's Laboratory Information System (CamLIS) was developed by the Ministry of Health in Cambodia with the support of WHO starting in 2011 (WHO, 2019). As of 2018, 35 national, provincial, and referral laboratories contribute to CamLIS. Cambodia has started to prepare the GLASS report for 2020, and the actual status of AMR bacteria in the country will be revealed in the near future. To date, however, only a few studies have examined AMR bacteria clinically isolated in Cambodia, although other Southeast Asian countries are increasingly reporting cases of AMR bacteria (Suwantarat and Carroll, 2016; Gandra et al., 2020). To date, there has been no report on the genomic epidemiology of CPGNB in Cambodia.

In this study, we introduced portable laboratory equipment, Bento Lab and MinION, for on-site genomic epidemiological analysis of CPGNB in Cambodia. Bento Lab (Bento Bioworks Ltd., United Kingdom) is a DNA analysis device small enough to fit in a laptop-sized bag. It contains a PCR thermal cycler, microcentrifuge, and gel electrophoresis apparatus with LED transilluminator, and has sufficient functionality for laboratory work (Bento Lab, 2016). The MinION nanopore sequencer (Oxford Nanopore Technologies, United Kingdom) is a portable long-read sequencer with the size of a large USB memory stick. MinION was utilized for on-site genomic epidemiological analysis of the Ebola virus outbreak in West Africa in 2016 (Quick et al., 2016) and the Zika virus outbreak in the Americas in 2017 (Faria et al., 2017). Because carbapenemase genes are mostly carried on plasmids, long-read sequencing is useful for assembling whole plasmid sequences and tracking horizontal transfer of AMR plasmids in hospitals, as well as local and global communities (Conlan et al., 2014).

We organized an international collaborative research group with researchers from Japan, United Kingdom, and Cambodia, and successfully performed on-site genomic epidemiological analysis of CPGNB clinical isolates in Cambodia, where access to laboratory equipment is limited. Our findings demonstrate the potential impact of portable laboratory equipment on AMR bacteria research and provide the first glimpse into the genomic epidemiology of CPGNB in Cambodia.

\section{MATERIALS AND METHODS}

\section{Ethics}

Written informed consent was obtained from the individuals for the publication of any potentially identifiable images included in this article.

\section{Subjects and Specimen Collection}

The outpatient clinic of National Institute of Public Health (NIPH) in Phnom Penh, Cambodia has around 10 patients in a day and 456 bacterial strains were isolated from patient specimens, such as sputum, stool, urine, pus, body fluid, and cerebral spinal fluid, in 2017. Ethical approval of this study "Genomic epidemiological analysis of AMR bacterial isolates in Cambodia" was obtained from National Ethics Committee for Health Research (NECHR), Cambodia (approval no.: 178NECHR). Two carbapenemase-producing isolates NIPH17_0020 and NIPH17_0036 of Escherichia coli and one carbapenemase-producing isolate NIPH17_0019 of Acinetobacter baumannii analyzed in this study were obtained from abdominal pus, urine, and blood of patients, respectively, at NIPH, Cambodia in 2017.

\section{Bacterial Isolates}

Bacterial species identification was performed using conventional biochemical tests (e.g., citrate test, urease test, hydrogen sulfide test, oxidase test, indole test, lysine decarboxylase test, and carbohydrate fermentation test) and the API 20E/20NE systems (bioMérieux), and antimicrobial susceptibility testing with E. coli ATCC 25922 as quality control was performed using BBL Sensi-Disc Susceptibility Test Disks (BD) as part of routine diagnosis at NIPH, Cambodia. Minimum inhibitory concentrations (MICs) of selected antimicrobials, including imipenem (IPM), meropenem (MEM), ceftazidime (CAZ), 
cefotaxime (CTX), aztreonam (AZT), amikacin (AMK), and ciprofloxacin (CPFX), against carbapenemase-producing isolates of E. coli (NIPH17_0020 and NIPH17_0036) and A. baumannii (NIPH17_0019) were further examined using the E-test strips (bioMérieux) in this study. The breakpoints for susceptible (S), intermediate (I), and resistance (R) to antimicrobials were adopted from the Clinical and Laboratory Standards Institute (CLSI) 2020 guidelines. Carbapenemase production was examined using the carbapenem inactivation method (CIM) according to the CLSI guidelines also as routine diagnosis at NIPH, Cambodia. The double-disk diffusion tests (DDDTs) with clavulanate (CVA) and sodium mercaptoacetic acid (SMA) as specific inhibitors for extended spectrum $\beta$-lactamases (ESBLs) and metallo- $\beta$-lactamases (MBLs), respectively, were performed as previously described (Arakawa et al., 2000; Hattori et al., 2013; CLSI, 2015). Briefly, the production of ESBLs was tested with the combination of CAZ, CTX, and clavulanate/amoxicillin (CVA/AMPC) disks (Eiken Chemical Co.), and production of MBLs was tested with the combination of IPM and SMA disks (Eiken Chemical Co.).

\section{PCR, Whole-Genome Sequencing, and Bioinformatics Analysis}

Draft genome analysis of carbapenemase-producing isolates of E. coli (NIPH17_0020 and NIPH17_0036) and A. baumannii (NIPH17_0019) using Bento Lab and MinION was performed in NIPH, Cambodia in July, 2017. Bacterial genomic DNAs (gDNAs) were extracted using the MagAttract HMW DNA Kit (Qiagen) and quantified using a Qubit 2.0 fluorometer (Thermo Fisher Scientific). Library preparation for MinION sequencing using Rapid Sequencing Kits (SQK-RAD002 and SQK-RAD003) (Oxford Nanopore Technologies) were performed using the prototype model of Bento Lab (Bento Bioworks Ltd.) consisting of a thermal cycler, microcentrifuge, and gel electrophoresis apparatus with LED transilluminator (Bento Lab, 2016). The prototype is configured slightly differently from the current commercial versions, but there is no significant difference in performance (Supplementary Figure 1).

PCR for selected carbapenemase genes, bla $a_{\mathrm{NDM}}$ (621-bp), $b l a_{\mathrm{KPC}}(798-\mathrm{bp}), b l a_{\mathrm{IMP}}(232-\mathrm{bp})$, bla $_{\mathrm{VIM}}$ (390-bp), bla $a_{\mathrm{OXA}-48}$ (438-bp), bla $a_{\mathrm{OXA}-23}$ (501-bp), bla $a_{\mathrm{OXA}-24}$ (246-bp), bla $a_{\mathrm{OXA}-51}$ (353-bp), and $b l a_{\mathrm{OXA}-58}$ (599-bp)] was performed using primers as previously described (Woodford et al., 2006; Poirel et al., 2011b). PCR amplification products were subjected to agarose gel electrophoresis using electrophoresis apparatus supplied with Bento Lab, stained with SYBR Gold Nucleic Acid Gel Stain (Thermo Fisher Scientific), detected using LED transilluminator built in Bento Lab, and photographed with iPhone 7 Plus (Apple).

Whole-genome sequencing was performed on the MinION nanopore sequencer (Oxford Nanopore Technologies) for $24 \mathrm{~h}$ with the offline-capable version of MinKNOW v1.7.3 and R9.4 flow cells according to the manufacturer's instructions. Nanopore reads were base called using Albacore v2.1.0 (Oxford Nanopore Technologies), corrected using Genome Finishing Module v1.7 plugged-in CLC Genomics Workbench v10.1.1 (Qiagen) with default parameters of Correct PacBio Reads (beta), and assembled de novo using Miniasm v0.2 (Li, 2016) with default parameters.

The extracted bacterial gDNAs were subsequently resequenced on a Illumina system in National Institute of Infectious Diseases, Japan for further error correction. Library for Illumina sequencing (insert size of 500-900 bp) was prepared using Nextera XT DNA Library Prep Kit (Illumina) and paired-end sequencing ( $2 \mathrm{bp} \times 150 \mathrm{bp})$ was performed using MiniSeq (Illumina). Illumina paired-end reads were mapped onto the on-site assembly sequences, and sequencing errors were corrected by extracting the consensus of the mapped reads five times using CLC Genomics Workbench v12.0 (Qiagen) with default parameters.

Genome sequences were annotated using the DFAST server ${ }^{1}$. Sequence type (ST), plasmid replicon type, AMR genes, and virulence genes were detected using MLST v2.0, PlasmidFinder v2.1, ResFinder v4.1, and VirulenceFinder v2.0, respectively, using the CGE server ${ }^{2}$ with default parameters. Type IV secretion system (T4SS)-associated genes involved in conjugation were detected using TXSScan ${ }^{3}$ with default parameters. Mobile gene elements (MGEs) were identified manually from CDS annotations and basically analyzed by comparing the sequences analyzed in previous studies. Linear comparisons of sequences carrying carbapenemase genes were performed using BLAST with default settings (the nucleotide collection database and the megablast program) and visualized using Easyfig v2.2.2 ${ }^{4}$. The annotated bacterial circular chromosomes were visualized using the CGView Server ${ }^{5}$.

Genome and plasmid sequences of carbapenemase-producing E. coli (NIPH17_0020 and NIPH17_0036) and A. baumannii (NIPH17_0019) isolated in Cambodia have been deposited at GenBank/EMBL/DDBJ under accession numbers AP024560 (NIPH17_0020), LC483178 (pNIPH17_0020_1), AP024561 (NIPH17_0036), LC483179 (pNIPH17_0036_1), LC603215 (pNIPH17_0036_2), and AP024415 (NIPH17_0019).

\section{RESULTS}

\section{On-Site Genomic Epidemiological Analysis of Carbapenemase-Producing Gram-Negative Bacteria Isolated in Cambodia}

In July 2017, we stayed for 5 days at the National Institute of Public Health (NIPH) in Phnom Penh, Cambodia. There, we set up potable laboratory equipment, including Bento Lab and MinION, in the Bacteriology laboratory, which has limited research facilities and no PCR machine (Figure 1). On the first and second days, we performed the carbapenem inactivation method (CIM) test, double-disk diffusion tests (DDDTs), minimum inhibitory concentrations (MICs) measurement,

\footnotetext{
${ }^{1}$ https://dfast.nig.ac.jp

${ }^{2}$ http://www.genomicepidemiology.org

${ }^{3}$ https://galaxy.pasteur.fr/

${ }^{4}$ http://mjsull.github.io/Easyfig/

${ }^{5}$ http://cgview.ca
} 


\section{Potable laboratory equipment in Bacteriology laboratory, National Institute of Public Health (NIPH), Phnom Penh, Cambodia}

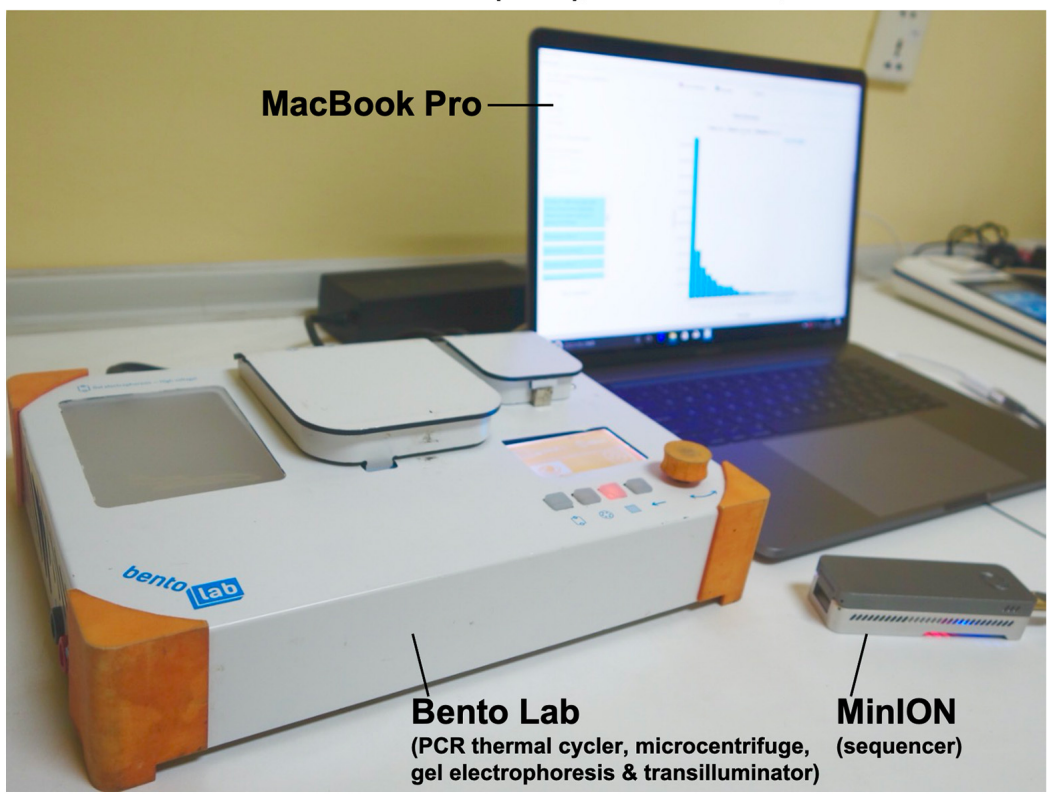

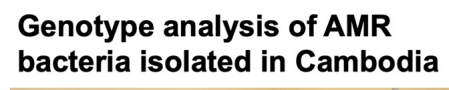

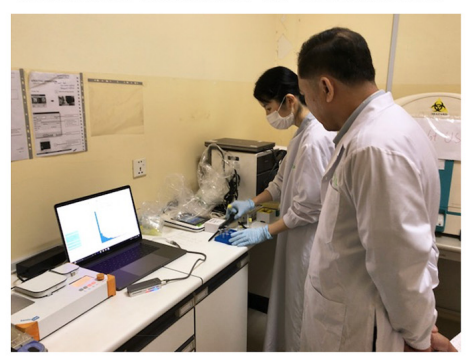

Phenotype analysis of AMR bacteria isolated in Cambodia

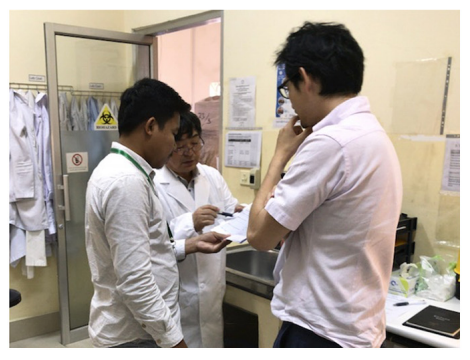

FIGURE 1 | On-site genomic epidemiological analysis of AMR bacteria in Cambodia. Bento Lab and MinION were used for genotype analysis and the carbapenem inactivation method (CIM) and double-disk diffusion tests (DDDTs) were used for phenotype analysis of AMR bacteria.

PCR, and MinION sequencing on two carbapenemaseproducing isolates of E. coli (NIPH17_0020 and NIPH17_0036) and one carbapenemase-producing isolate of $A$. baumannii (NIPH17_0019) (Figure 1) stored in the laboratory prior to this study. On the third and fourth days, we examined diagnostic testing data (Figure 2) and analyzed sequencing data (Figures 35). On the final day, we discussed the results of genotype and phenotype analysis with researchers and technicians belonging to the Bacteriology laboratory at NIPH.

The CIM test is routinely performed at NIPH, and we confirmed that all three bacterial isolates were positive for carbapenemase production. DDDTs with sodium mercaptoacetic acid (SMA) and clavulanic acid (CVA) disks revealed that E. coli NIPH17_0020 and E. coli NIPH17_0036 were positive for MBL and ESBL production, respectively (Figure 2A). PCR targeting major carbapenemase genes revealed that E. coli NIPH17_0020, E. coli NIPH17_0036, and A. baumannii NIPH17_0019 were positive for $b l a_{\mathrm{NDM}}, b l a_{\mathrm{OXA}-48}$, and $b l a_{\mathrm{OXA}-23}$, respectively (Figure 2B). The MICs of imipenem (IPM) and meropenem (MEM) against E. coli NIPH17_0020, E. coli NIPH17_0036, and A. baumannii NIPH17_0019 were 2 (I) and 2 (I), 0.38 (S) and $0.25(\mathrm{~S})$, and $>32(\mathrm{R})$ and $>32 \mu \mathrm{g} / \mathrm{mL}(\mathrm{R})$, respectively. Furthermore, the MICs of ceftazidime (CAZ), cefotaxime (CTX), aztreonam (AZT), amikacin (AMK), and ciprofloxacin (CPFX) against E. coli NIPH17_0020 were >256 (R), >32 (R), 4 (S), $1(\mathrm{~S})$, and $>32 \mu \mathrm{g} / \mathrm{mL}(\mathrm{R})$, respectively; those against $E$. coli NIPH17_0036 were > $256(\mathrm{R}),>32(\mathrm{R}),>256(\mathrm{R}), 2$ (S), and $>32 \mu \mathrm{g} / \mathrm{mL}(\mathrm{R})$, respectively; and those against A. baumannii NIPH17_0019 were > $256(\mathrm{R}),>32(\mathrm{R}),>256,>256(\mathrm{R})$, and $>32 \mu \mathrm{g} / \mathrm{mL}(\mathrm{R})$, respectively (Figure $2 \mathrm{C}$ ).
Based on the on-site de novo assembly sequences obtained from nanopore sequencing analysis, we determined the complete structures of chromosomes and plasmids of each bacterial isolate and detected AMR genes. As shown in Supplementary Table 1, E. coli NIPH17_0020 had two contigs (the 4.78-Mb chromosome and the 91.6-kb plasmid pNIPH17_0020_1); E. coli NIPH17_0036 had three contigs (the 4.68-Mb chromosome, the 50.2-kb plasmid pNIPH17_0036_1, and the 92.6-kb plasmid pNIPH17_0036_2); and A. baumannii NIPH17_0019 had only one contig (the 3.85-Mb chromosome). E. coli pNIPH17_0020_1 carried the bla $a_{\mathrm{NDM}-5}$-like gene $(96.3 \%$ identity and $3.0 \%$ gap relative to bla $a_{\mathrm{NDM}-5}$ : accession no. JN104597) (Supplementary Figure 2A); E. coli pNIPH17_0036_1 carried the bla OxA-48 family carbapenemase $b l a_{\text {OXA }}-181$-like gene $(97.2 \%$ identity and $2.5 \%$ gap relative to $b l a_{\mathrm{OXA}-181}$ : accession no. CM004561) (Supplementary Figure 2B); and A. baumannii NIPH17_0019 harbored bla $a_{\mathrm{OXA}-23}$-like genes in two separate locations of the chromosome $(97.7 \%$ identity and $2.1 \%$ gap or 93.9 and $5.6 \%$ gap relative to bla $a_{\mathrm{OXA}-23}$ : accession no. AY795964) (Supplementary Figure 2C).

To summarize the results, the detected carbapenemase genes had a few-percent mismatch that caused frameshifts of genes relative to their putative reference sequences (Supplementary Figure 2); hence, we avoided performing CDS annotation for the on-site sequences. We detected AMR genes and plasmid replicons in the sequences analyzed on-site by sequencebased detection (Figures 3-5 and Supplementary Table 1). AMR genes, such as bla ${ }_{\mathrm{TEM}-1 \mathrm{~B}}$, aadA2, $a p h\left(3^{\prime \prime}\right)-l b, \operatorname{aac}(3)-$ $l l d$, and $a p h(6)-l d$, and plasmid replicons, including IncFIA, IncFIB, IncFII, and IncQ1, were detected in provisional 
A

Double-disk diffusion tests (DDDTs)

MBL detection using SMA

E. coli NIPH17_0020

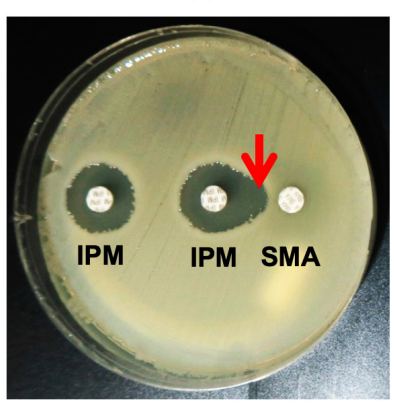

ESBL detection using CVA E. coli NIPH17_0036

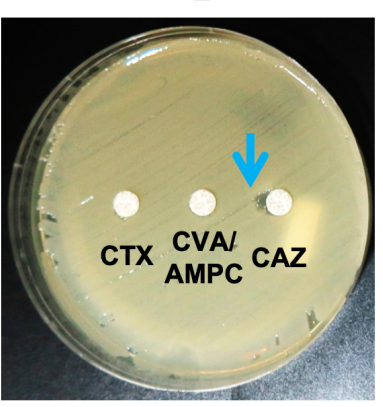

B

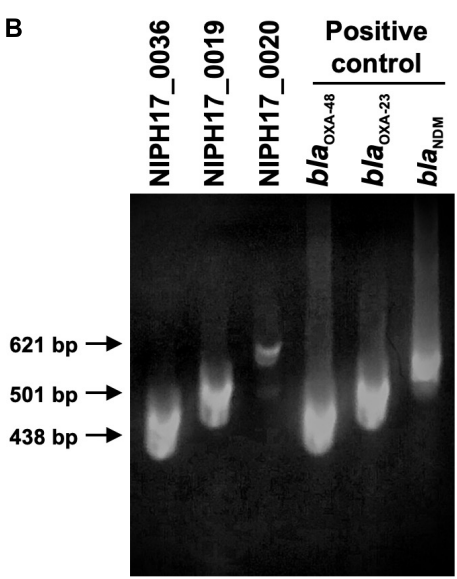

C

\begin{tabular}{|c|c|c|c|c|c|c|c|c|c|c|c|}
\hline \multirow{2}{*}{ Isolate } & \multirow{2}{*}{ CIM } & \multicolumn{3}{|c|}{ DDDT } & \multicolumn{6}{|c|}{ MIC ( $\mu \mathrm{g} / \mathrm{ml})$} & \multirow{2}{*}{ PCR } \\
\hline & & SMA & CVA & IPM & MEM & CAZ & CTX & AZT & AMK & CPFX & \\
\hline $\begin{array}{l}\text { E. coli } \\
\text { NIPH17_0020 }\end{array}$ & Posi. & Posi. & Neg. & $\begin{array}{l}2 \\
\text { (I) }\end{array}$ & $\begin{array}{l}2 \\
\text { (I) }\end{array}$ & $\begin{array}{l}>256 \\
(R)\end{array}$ & $\begin{array}{l}>32 \\
(\mathrm{R})\end{array}$ & $\begin{array}{c}4 \\
\text { (S) }\end{array}$ & $\begin{array}{l}1 \\
(S)\end{array}$ & $\begin{array}{l}>32 \\
\text { (R) }\end{array}$ & $b / a_{\text {NDM }}$ \\
\hline $\begin{array}{l}\text { E. coli } \\
\text { NIPH17_0036 }\end{array}$ & Posi. & Neg. & Posi. & $\begin{array}{c}0.38 \\
\text { (S) }\end{array}$ & $\begin{array}{c}0.25 \\
\text { (S) }\end{array}$ & $\begin{array}{c}>256 \\
(R)\end{array}$ & $\begin{array}{c}>32 \\
(R)\end{array}$ & $\begin{array}{c}>256 \\
\text { (R) }\end{array}$ & $\begin{array}{l}2 \\
\text { (S) }\end{array}$ & $\begin{array}{c}>32 \\
(\mathrm{R})\end{array}$ & $b / a_{0 \times A-48}$ \\
\hline $\begin{array}{l}\text { A. baumannii } \\
\text { NIPH17_0019 }\end{array}$ & Posi. & Neg. & Neg. & $\begin{array}{c}>32 \\
(R)\end{array}$ & $\begin{array}{c}>32 \\
(\mathrm{R})\end{array}$ & $\begin{array}{l}>256 \\
\text { (R) }\end{array}$ & $\begin{array}{c}>32 \\
(R)\end{array}$ & $>256$ & $\begin{array}{l}>256 \\
\text { (R) }\end{array}$ & $\begin{array}{l}>32 \\
(\mathrm{R})\end{array}$ & $b / a_{0 \times A-23}$ \\
\hline
\end{tabular}

FIGURE 2 | Genotype and phenotype analysis of AMR bacteria isolated in Cambodia. (A) The double-disk diffusion test (DDDT) with imipenem (IPM) and sodium mercaptoacetic acid (SMA) disks against E. coli NIPH17_0020 for MBL detection and DDDT with ceftazidime (CAZ), cefotaxime (CTX), and clavulanate/amoxicillin (CVAAMPC) disks against E. coli NIPH17_0036 for ESBL detection. Arrows indicate $\beta$-lactamase inhibition. (B) PCR amplifications for blaOXA-48 for E. coli NIPH17_0036 (438 bp), blaOXA-23 for A. baumannii NIPH17_0019 (501 bp), blanDM for E. coli NIPH17_0020 (621 bp) and their positive controls. Some of the images are unclear because they were acquired in a location with limited equipment. (C) Summary of genotype and phenotype analysis, including the carbapenem inactivation method (CIM), DDDTs with SMA or CVA, minimum inhibitory concentrations (MIC) measurement, and PCR targeting selected major carbapenemase genes. The breakpoints for susceptible (S), intermediate (I), and resistance (R) to antimicrobials were adopted from the CLSI 2020 guidelines.

bla $a_{\mathrm{NDM}-5}$-carrying pNIPH17_0020_1 in E. coli NIPH17_0020 (Figure 3), and qnrS1 genes and the IncX3 replicon were detected in provisional bla 0 A-181-carrying pNIPH17_0036_1 in E. coli NIPH17_0036 (Figure 4). BLAST searches of E. coli pNIPH17_0020_1 and E. coli pNIPH17_0036_1 revealed that several plasmids from Asian and Western countries were highly identical to those plasmids (Figures 3, 4).

\section{Comparison of Plasmids and Genomic Regions in Carbapenemase-Producing Gram-Negative Bacteria Isolated in Cambodia With Those in Other Countries}

After the on-site analysis in Cambodia, we further performed Illumina sequencing of carbapenemase-producing isolates of E. coli (NIPH17_0020 and NIPH17_0036) and A. baumannii (NIPH17_0019), corrected the on-site de novo assembly sequences using Illumina reads, and compared the onsite and error-corrected sequences (Figures 3, 4, 5A, and Supplementary Figure 3 ). The on-site sequences of E. coli pNIPH17_0020_1 (provisional 91.6-kb bla $a_{\mathrm{NDM}-5 \text {-carrying }}$ plasmid with IncFIA/FIB/FII/Q1 replicons) and $E$. coli pNIPH17_0036_1 (provisional 50.2-kb bla OXA-181-carrying plasmid with IncX3 replicons) were highly identical to their error-corrected sequences (96.48\% identity over $100 \%$ of the error-corrected 93.9-kb plasmid pNIPH17_0020_1: accession no. LC483178 and $96.70 \%$ identity over $100 \%$ of the error-corrected plasmid 51.5-kb pNIPH17_0036_1: accession no. LC483179, respectively) (Figures 3,4). Moreover, the on-site sequences of

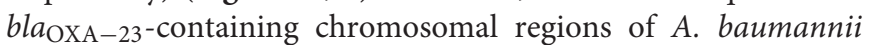
NIPH17_0019 were also highly identical to their error-corrected sequences (96.95\% identity over $100 \%$ of $216,633-233,441 \mathrm{nt}$ and $97.36 \%$ identity over $100 \%$ of $3,924,674-3,941,483 \mathrm{nt}$ in their error-corrected chromosome of NIPH17_0019: accession no. AP024415) (Supplementary Figures 3A,B). Although there were differences of a few percent between the on-site and error-corrected sequences, the best match types of AMR genes and plasmid replicons detected from the reference libraries were consistent (Figures 3, 4, 5A).

Escherichia coli NIPH17_0020 belonged to sequence type 410 (ST410) according to multilocus sequence typing (MLST) analysis and harbored putative virulence genes, 


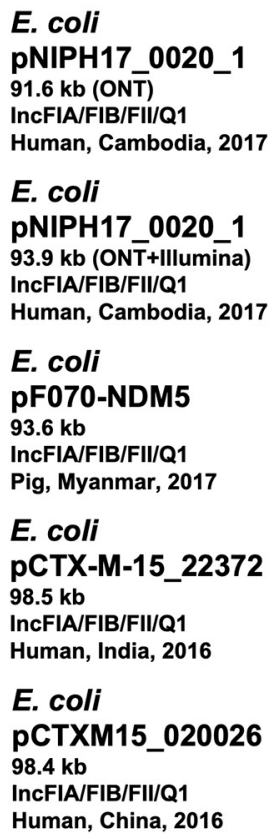

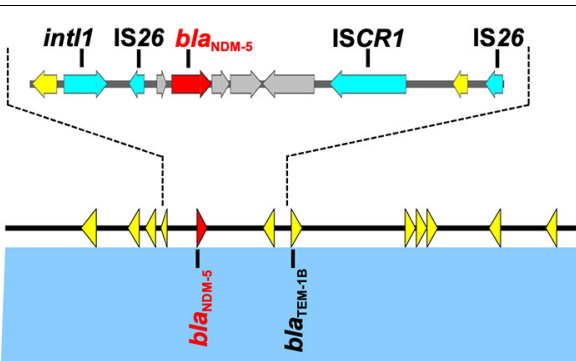
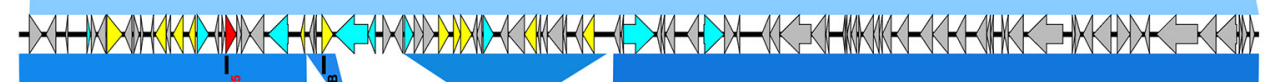
紊

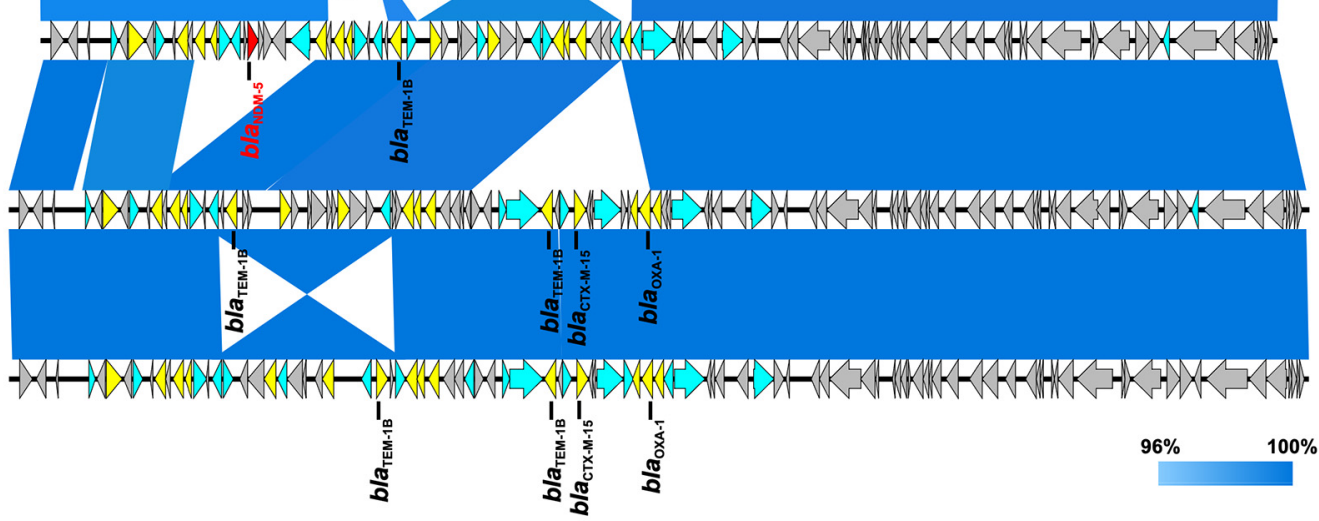

bla $_{\mathrm{OXA-181}} \square$ ARG
T4SS
MGE

Others

FIGURE 3 | Linear comparison of related plasmid sequences from Cambodia and other countries. E. coli pNIPH17_0020_1 with bla LC483178), E. coli pF070-NDM5 with bla NDM-5 (accession no. AP023238), E. coli pCTX-M-15_22372 with no blandM (accession no. CP040398), and E. coli pCTXM15_020026 with no blandM (accession no. CP034956) are shown. Regarding E. coli pNIPH17_0020_1, both the uncorrected sequence (from on-site ONT analysis) and corrected sequence (from subsequent ONT + Illumina analysis), and the detailed genetic structures around blanDM-5 are shown (upper). Red, yellow, green, blue, and gray arrows indicate carbapenemase gene (bla NDM-5), other AMR genes (ARG), type IV secretion system-associated genes involved in conjugation (T4SS), mobile gene elements (MGE), and other genes (Others), respectively. The colors in comparison of plasmids show percent identity.

including $f y u A$, gad, irp2, lpfA, and terC on its chromosome (Supplementary Table 1). NIPH17_0020 had one 93.9-kb plasmid, pNIPH17_0020_1, with a backbone consisting of IncFIA/FIB/FII/Q1 with multiple AMR genes, such as $\beta$ lactamase $\left(b l a_{\mathrm{NDM}-5}\right.$ and $\left.b l a_{\mathrm{TEM}-1 \mathrm{~B}}\right)$ and aminoglycoside resistance genes [aac(3)-lld-like, aadA2, aph(3")-lb, aph(6)ld] (Supplementary Table 1). E. coli pNIPH17_0020_1 was structurally highly identical to plasmid pPF070-NDM5 (accession no. AP023238) in E. coli isolated from a pig in Myanmar in 2017, plasmid pCTX-M-15_22372 (accession no. CP040398) in E. coli isolated from a human in India in 2016, and plasmid pCTXM15_020026 (accession no. CP034956) in E. coli isolated from a human in China in 2016 (99.8\% identity over 92-94\% of pNIPH17_0020_1) (Figure 3). pNIPH17_0020_1 contained several mobile gene elements (MGEs), including IS26 and ISCR1, surrounding bla $a_{\mathrm{NDM}-5}$ (Figure 3 upper). pF070-NDM5 carried bla $a_{\mathrm{NDM}-5}$ with the same MGEs, whereas pCTX-M-15_22372 and pCTXM15_020026 did not carry bla $a_{\mathrm{NDM}-5}$ (Figure 3).

Escherichia coli NIPH17_0036 also belonged to ST410 according to MLST analysis and harbored putative virulence genes, including gad, hra, $\operatorname{lpfA}$, and terC on its chromosome (Supplementary Table 1). NIPH17_0036 had two plasmids; one of them, 51.5-kb IncX3 plasmid pNIPH17_0036_1 carrying AMR genes, including bla $a_{\mathrm{OXA}-181}$ and qnrS1 (Supplementary Table 1), was structurally nearly identical to plasmid pOXA181-IHIT35346 (accession no. KX894452) in E. coli isolated from a pig in Italy in 2016 and plasmid pOXA181 (accession no. KP400525) in E. coli isolated from a human in China in 2014 (Liu et al., 2015), as well as plasmid pKS22 (accession no. KT005457) in Klebsiella variicola isolated from a fresh vegetable imported from Thailand or Vietnam (99.9\% identity over $100 \%$ of pNIPH17_0036_1) (Figure 4). bla $\mathrm{OXA}-181$ in pNIPH17_0036_1 was surrounded by several MGEs, including ISKpn19, IS3000, and IS26 (Figure 4 upper). E. coli NIPH17_0036 had another plasmid, pNIPH17_0036_2 (94.8-kb IncFIA/FIB/FII/Q1 plasmid, accession no. LC603215), carrying bla $a_{\mathrm{CTX}-\mathrm{M}-15}$, bla $a_{\mathrm{OXA}-1}, b l a_{\mathrm{TEM}-1 \mathrm{~B}}$, other $\beta$-lactamase genes, and multiple aminoglycoside resistance genes (Supplementary Table 1).

Acinetobacter baumannii NIPH17_0019 belonged to ST471 according to MLST analysis and harbored the bla OXA-23 genes in two separate regions on the chromosome (accession no. 


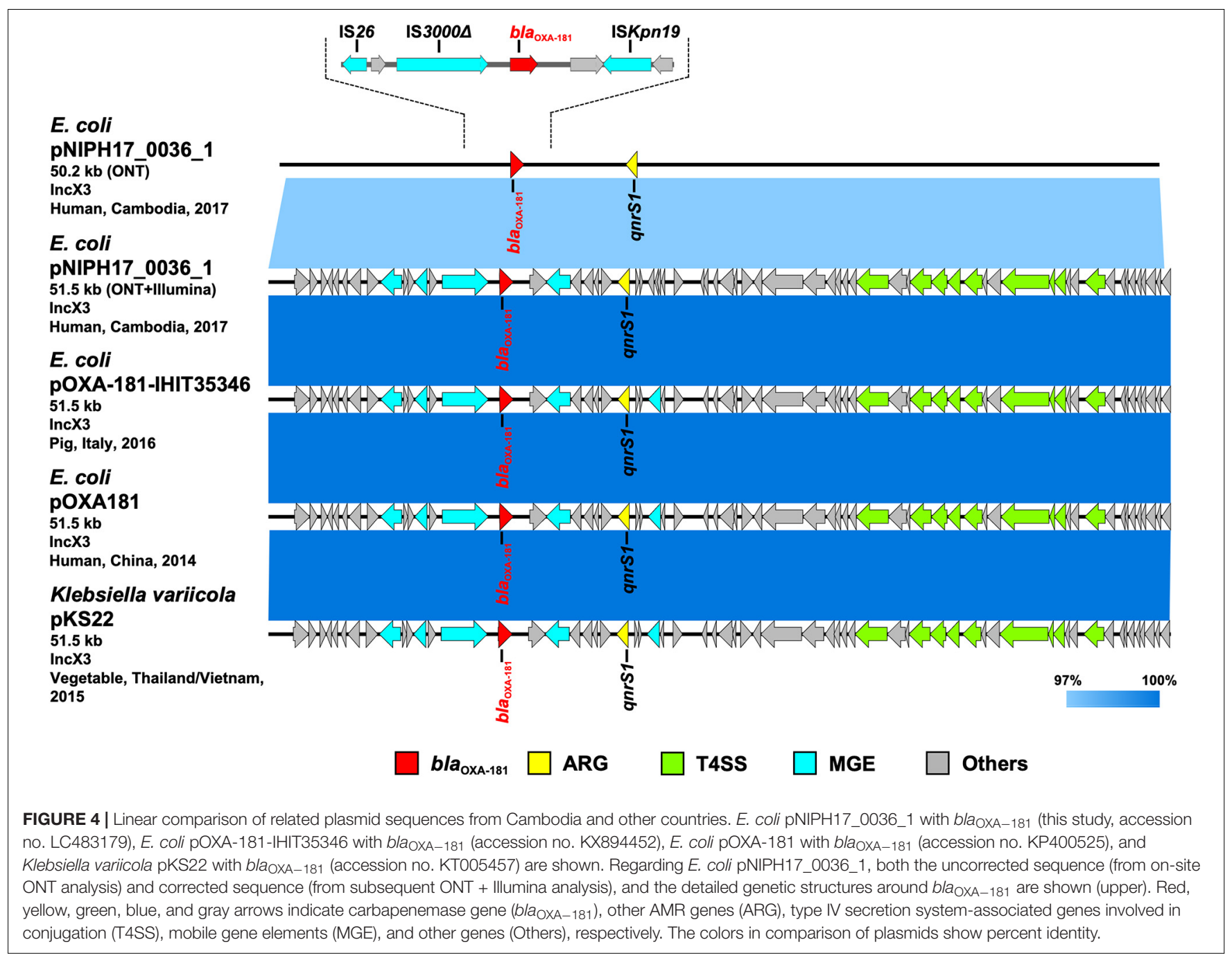

AP024415) (Figure 5A). Both copies of bla $a_{\mathrm{OXA}-23}$ were located in Tn2006 in AbaR4. AbaR is the resistance island containing AMR genes in A. baumannii (Bi et al., 2019). Comparison of the genetic environment around AbaR4 was performed between A. baumannii NIPH17_0019 and AbaR4-harboring A. baumannii D36 (accession no. JN107991), which was isolated from a human in 2008 in Australia (Figure 5B). The results revealed that two AbaR4 islands in A. baumannii NIPH17_0019 were highly identical to that of A. baumannii D36 (99.84-99.88\% identity over $100 \%$ of the sequence) (Figure 5B). Interestingly, $\mathrm{AbaR} 4$ was integrated into the comM gene in A. baumannii D36, whereas AbaR4 was integrated into the comM gene (AbaR4 of the 216,633-233,441 nt region) and mutY genes (for AbaR4 of the 3,924,674-3,941,483 nt region) in A. baumannii NIPH17_0019.

\section{DISCUSSION}

In this study, we successfully conducted genomic analysis of carbapenemase-producing gram-negative bacteria (CPGNB) in Cambodia and provided the first glimpse into the genomic epidemiology of CPGNB in that country. The main analysis was performed on-site using portable laboratory equipment, namely, Bento Lab and MinION. The nearly complete genomes of carbapenemase-producing E. coli and A. baumannii isolates, including their plasmids, were determined using the MinION nanopore sequencing data; detection of AMR genes and plasmid replicons, as well as sequence similarity searches in public databases, were performed at a Cambodian laboratory with limited research facilities. Because the nanopore sequencing technology is still evolving, and the accuracy of sequencing is imperfect (the rates of indels and substitutions are a few percent each) (Jain et al., 2016), it was necessary to combine other sequencing technologies, such as Illumina sequencing by synthesis, for further molecular typing analysis of bacteria that require more accurate sequences at the single-nucleotide level.

The cost of commercial versions of Bento Lab is starting from $\$ 1,646.99^{6}$, whereas MinION is free of charge because it is basically a rental from the company ${ }^{7}$. In this study, we

\footnotetext{
${ }^{6}$ https://us.vwr.com/store/product/27856428/bento-lab

${ }^{7}$ https://store.nanoporetech.com/configure-minion-basic
} 

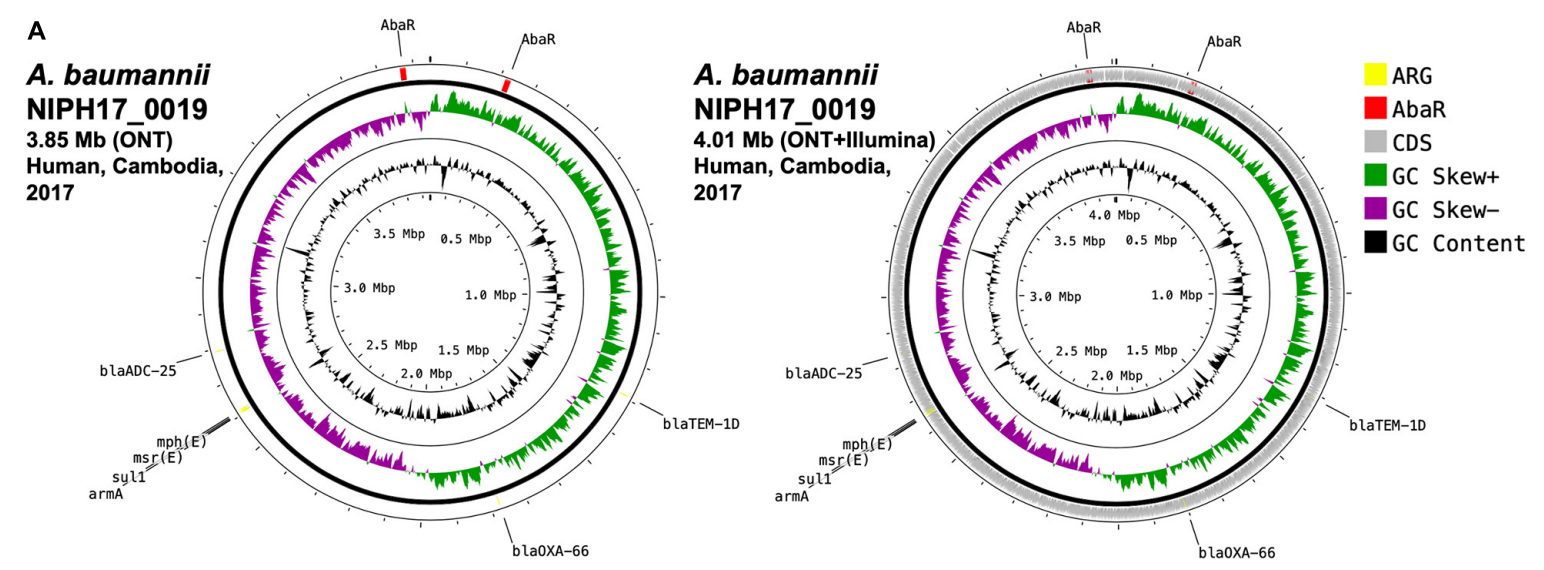

B

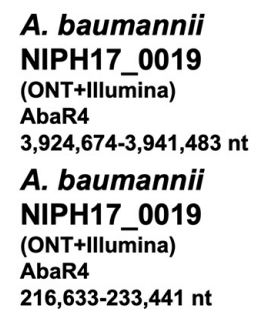

A. baumannii D36

Human, Australia, 2008

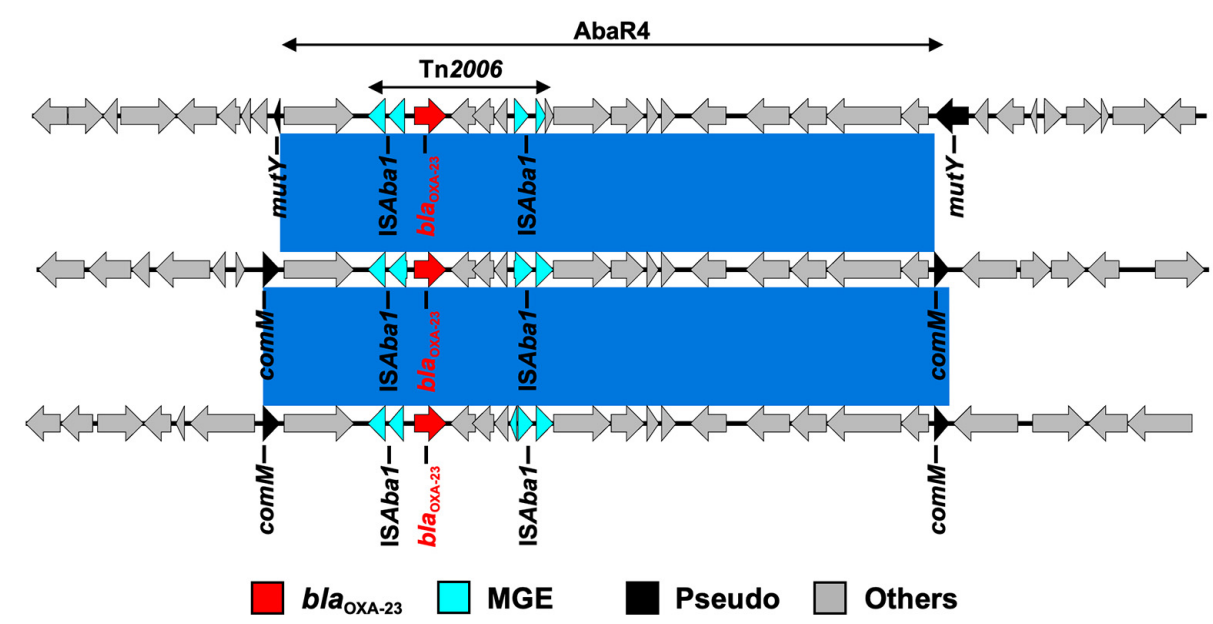

FIGURE 5 | (A) Circular chromosome representation of A. baumannii NIPH17_0019 harboring two copies of bla_XA-23. Both the uncorrected sequence (from on-site ONT analysis) and corrected sequence (from subsequent ONT + llumina analysis, accession no. AP024415) are shown. Yellow, red, gray, green, purple, and black indicate AMR genes (ARG), AbaR4 with blaOXA-23 (AbaR), other coding sequences (CDS), GC skew+, GC skew-, and GC content, respectively. (B) Linear comparison of AbaR4-containing genomic regions in A. baumannii from Cambodia and other countries. Two sets of AbaR4 with blaoxA-23 in A. baumannii NIPH17_0019 (3,924,674-3,941,483 nt region inserted within mutY and 216,633-233,441 nt region inserted within comM in accession no. AP024415) and AbaR4 with blaOXA-23 in A. baumannii D36 (41,312-58,123 nt region inserted within comM in accession no. JN107991) are shown. Red, blue, black, and gray arrows indicate carbapenemase gene (bla OXA-23), mobile gene elements (MGE), pseudogenes disrupted by AbaR4 insertions (Pseudo), and other genes (Others), respectively. The blue color in comparison of sequences indicates nearly $100 \%$ identity.

did not use barcodes for MinION library preparation and performed MinION analysis using one flow cell per sample to ensure reliable data acquisition. The cost of the flow cell and library preparation reagent was $\$ 599$ (for 6 reactions) and $\$ 900$, respectively, resulting in MinION analysis cost of $\$ 999$ per sample. If barcodes are used for library preparation, the unit cost of MinION analysis can be dramatically reduced. We usually analyze about six barcoded samples using one flow cell for bacterial species with genome sizes of around $5 \mathrm{Mb}$. If six samples are included in one analysis, the unit cost is going down to $\$ 171^{8}$.

The carbapenem inactivation method (CIM) and doubledisk diffusion test (DDDT) are simple, inexpensive, and useful, especially in developing countries where materials and facilities are limited. In this study, the results of both tests were reasonable,

\footnotetext{
${ }^{8}$ https://store.nanoporetech.com/catalog/product/view/id/226/s/rapidbarcoding-kit/category/28/
}

as validated by subsequent genetic analysis (Figure 2). The CIM test is routinely performed at NIPH, Cambodia, and NIPH had detected and stored carbapenemase-producing bacterial isolates prior to this study. The DDDTs with SMA and CVA disks clearly detected production of MBL in E. coli NIPH17_0020 and ESBL in E. coli NIPH17_0036 (Figure 2A). CIM and DDDT are important for screening for CPGNB because MICs of carbapenems against CPGNB are not always high, as is the case for bacteria with carbapenem-hydrolyzing oxacillinase genes, such as bla $a_{\mathrm{OXA}-48}$ or its variants. The MICs of carbapenems against E. coli NIPH17_0036, which harbored bla OXA-181 on its plasmid (Figure 4), were relatively low, whereas CIM yielded a positive result (Figure 2C). OXA-48 is capable of weakly hydrolyzing carbapenems while maintaining its activity against broad-spectrum cephalosporins. The bla $\mathrm{OXA}-181$ gene is a variant of bla OXA-48, and the hydrolytic activity of OXA-181 toward $\beta$-lactams is similar to that of OXA-48 (Castanheira et al., 2011). 
We sequenced carbapenemase-producing $E$. coli and A. baumannii isolates on-site using MinION and Bento Lab, and revealed that E. coli NIPH17_0020 and E. coli NIPH17_0036 harbored bla $a_{\mathrm{NDM}-5}$ and bla $a_{\mathrm{OXA}-181}$ on plasmids pNIPH17_0020_1 and pNIPH17_0036_1, respectively (Figures 3, 4) and that A. baumannii NIPH17_0019 harbored two copies of bla $a_{\mathrm{OXA}-23}$ on its chromosome (Figure 5). Although the MinION control software MinKNOW requires a constant internet connection, the company provided us with an offlinecapable version of MinKNOW. For de novo assembly using nanopore long-read data, we used the Miniasm software, which is fast and computationally inexpensive ( $\mathrm{Li}, 2016$ ). Because Miniasm assembles without error correction, MinION reads were error-corrected using the CLC Genomics Workbench pipeline for long-read sequencers prior to de novo assembly. The resultant assembly sequences obtained from on-site analysis still contained a few percent of errors that are responsible for gene frameshifts. However, the on-site de novo assembly sequences were sufficient for subsequent molecular epidemiological analysis (e.g., detection of AMR genes and genomic locations where the genes are encoded) (Figures 3-5 and Supplementary Figures 2, 3), and the error-corrected sequences using Illumina sequencing were subsequently used to confirm the results of the on-site analysis (Figures 3-5).

Escherichia coli isolates NIPH17_0020 and NIPH17_0036 belonged to ST410 according to MLST analysis. ST410 is a high-risk clone associated with AMR and recently emerged in among humans and the environment in Southeast Asia (Nadimpalli et al., 2019). In this study, both E. coli isolates harbored carbapenemase genes, $b l a_{\mathrm{NDM}-5}$ and $b l a_{\mathrm{OXA}-181}$, respectively, on their plasmids, and commonly harbored putative virulence genes, including gad (a glutamate decarboxylase gene), lpfA (a long polar fimbriae gene), and terC (a tellurium ion resistance gene) on their chromosomes (Supplementary Table 1). Furthermore, NIPH17_0020 harbored other virulence genes, fyuA (a siderophore receptor gene) and irp2 (a siderophore gene), and NIPH17_0036 harbored other virulence gene hra (a heat-resistant agglutinin gene) on their chromosomes (Supplementary Table 1).

Escherichia coli pNIPH17_0020_1 [93.9-kb IncFIA/FIB/FII/Q1 plasmid with bla $a_{\mathrm{NDM}-5}$, accession no. LC483179] was structurally highly identical to E. coli pPF070NDM5 in Myanmar (accession no. AP023238) (Figure 3) and harbored several mobile gene elements (MGEs), including IS26 and ISCR1 surrounding bla $a_{\mathrm{NDM}-5}$ (Figure 3 upper). The bla $a_{\mathrm{NDM}-5}$-containing regions between IS26 and ISCR1 in pNIPH17_0020_1 and pPF070-NDM5 were identical with those of IncFII plasmids, such as E. coli pM109_FII in Myanmar (accession no. AP018139), E. coli pGUE-NDM in France (accession no. JQ364967), and K. pneumoniae pCC1409-1 in South Korea (accession no. KT725789), implying that $b l a_{\mathrm{NDM}-5}$ was disseminated via plasmids and MGEs, such IS26, among Enterobacterales around the world (Sugawara et al., 2017).

Escherichia coli pNIPH17_0036_1 [51.5-kb IncX3 plasmid with bla $a_{\text {OXA-181 }}$, accession no. LC483179] was structurally nearly identical to E. coli pOXA-181-IHIT35346 from Italy (accession no. KX894452), E. coli pOXA181 from China (accession no.
KP400525), and $K$. variicola pKS22 from Thailand/Vietnam (accession no. KT005457) (Figure 4). Our analysis revealed

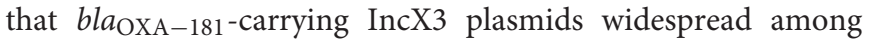
Enterobacterales worldwide were also present in Cambodia. pKS22 was detected in coriander imported from Thailand or Vietnam, and the international fresh vegetable trade is suspected to be a route for the spread of AMR bacteria (Zurfluh et al., 2015). Because Cambodia is geographically and culturally close to Thailand and Vietnam, it is possible for AMR bacteria to be transmitted through foods. However, this study was very small, so further analysis with larger numbers of bacterial isolates in Cambodia according to One Health approaches will be necessary to characterize AMR bacteria in this country.

Acinetobacter baumannii NIPH17_0019 belonging to ST571 harbored two copies of bla $a_{\mathrm{OXA}-23}$ in the chromosome (Figure 5A). According to a previous study of carbapenemresistant A. baumannii (Hamidian and Nigro, 2019), ST2 is the most prevalent genotype and recognized as a highrisk clone associated with $\mathrm{AMR}$, and bla $\mathrm{OXA}-23$ is the most widespread carbapenem-resistance gene in the world. ST571 belongs to clonal complex 2, and ST571 strains harboring $b l a_{\mathrm{OXA}-23}$ are widespread in medical settings in Vietnam (Tada et al., 2015). In general, carbapenem-hydrolyzing oxacillinases hydrolyze carbapenems weakly and do not contribute to strong carbapenem resistance on their own. However, elevated expression of oxacillinase genes by the upstream insertion of IS, such as ISAbal in Acinetobacter spp., which serves as a promoter for the downstream genes, leads to strong resistance (Turton et al., 2006; Corvec et al., 2007). Two copies of bla $a_{\mathrm{OXA}-23}$ in A. baumannii NIPH17_0019 were located downstream of ISAbal (Figure 5B). Both bla $a_{\mathrm{oxa}-23}$ were present in Tn2006, a common 4.8-kb transposon in Acinetobacter app., with a central segment of 2,445-bp flanked by two reverse-oriented copies of ISAba1 (Nigro and Hall, 2016). Tn2006-containing AbaR4 is frequently inserted within the comM gene in the chromosome of international A. baumannii clones (Hamidian and Hall, 2011; Seputiene et al., 2012; Hamidian et al., 2015), and the comM gene is the mostpreferred hotspot for AbaR insertions (Bi et al., 2019). One of the insertion sites of AbaR4 (3,924,674-3,941,483 nt region) in A. baumannii NIPH17_0019 was not the comM gene but the mutY gene, encoding for an adenine DNA glycosylase (Figure 5B). According to a previous study (Bi et al., 2019), AbaR insertions at acoA, pho, and uup genes were occasionally observed; however, mut $Y$ has not been previously reported as an insertion site.

\section{CONCLUSION}

Based on our on-site genomic epidemiological analysis of carbapenemase-producing gram-negative bacteria in Cambodia, we revealed for the first time that plasmids and MGEs carrying clinically relevant carbapenemase genes reported in other countries have also been spreading in Cambodia. Bento Lab and MinION are useful for genomic analysis and surveillance of AMR bacteria in hospitals and research centers with limited facilities. 


\section{DATA AVAILABILITY STATEMENT}

The datasets presented in this study can be found in online repositories. The names of the repository/repositories and accession number(s) can be found in the article/Supplementary Material.

\section{ETHICS STATEMENT}

The studies involving human participants were reviewed and approved by National Ethics Committee for Health Research (NECHR), Cambodia. The patients/participants provided their written informed consent to participate in this study. Written informed consent was obtained from the individuals for the publication of any potentially identifiable images included in this article.

\section{AUTHOR CONTRIBUTIONS}

MS contributed to conceptualization. AH, HY, and MS contributed to methodology. MS contributed to software. AH, HY, HT, KY, and MS contributed to validation. AH and MS contributed to formal analysis, data curation, and visualization. $\mathrm{AH}, \mathrm{HY}, \mathrm{HT}, \mathrm{KY}, \mathrm{KS}$, and MS contributed to investigation. $\mathrm{PB}$, $\mathrm{BW}, \mathrm{VN}, \mathrm{VL}, \mathrm{MV}, \mathrm{VA}$, and $\mathrm{CD}$ contributed to resources. AH, $\mathrm{KS}$, and MS contributed to writing. $\mathrm{CD}$ and KS contributed to supervision. $\mathrm{CD}, \mathrm{KS}$, and MS contributed to project administration. KS and MS contributed to funding. All authors contributed to the article and approved the submitted version.

\section{FUNDING}

This work was supported by Grants-in-Aid for the Research Program on Emerging and Re-emerging Infectious Diseases (JP20fk0108093, JP20fk0108139, JP20fk0108133, and JP20wm0225008 for MS and JP20fk0108061 for KS) and the Japan Initiative for Global Research Network on Infectious Diseases (J-GRID) (JP18fm0108003 and JP19fm0108006 for

\section{REFERENCES}

Arakawa, Y., Shibata, N., Shibayama, K., Kurokawa, H., Yagi, T., Fujiwara, H., et al. (2000). Convenient test for screening metallo-beta-lactamase-producing gram-negative bacteria by using thiol compounds. J. Clin. Microbiol. 38, 40-43.

Bento Lab. (2016). Nat. Biotechnol. 34:455. doi: 10.1038/nbt0516-455

Bi, D., Xie, R., Zheng, J., Yang, H., Zhu, X., Ou, H. Y., et al. (2019). Large-Scale Identification of AbaR-type genomic islands in Acinetobacter baumannii reveals diverse insertion sites and clonal lineage-specific antimicrobial resistance gene profiles. Antimicrob. Agents Chemother. 63:e02526-18.

CLSI (2015). Performance Standards for Antimicrobial Disk Susceptibility Tests, Twelfth Edn. Wayne, PA: CLSI.

Castanheira, M., Deshpande, L. M., Mathai, D., Bell, J. M., Jones, R. N., and Mendes, R. E. (2011). Early dissemination of NDM-1- and OXA-181-producing Enterobacteriaceae in Indian hospitals: report from the SENTRY Antimicrobial Surveillance Program, 2006-2007. Antimicrob. Agents Chemother. 55, 12741278. doi: $10.1128 /$ aac. $01497-10$
KS) from the Japan Agency for Medical Research and Development (AMED). This work was also supported by the Cooperative Research Grant from Research Center on Tropical Disease, Institute of Tropical Medicine, Nagasaki University (2019-Ippan-20).

\section{SUPPLEMENTARY MATERIAL}

The Supplementary Material for this article can be found online at: https://www.frontiersin.org/articles/10.3389/fmicb. 2021.675463/full\#supplementary-material

Supplementary Figure 1 | Bento Lab for on-site genomic epidemiological analysis of AMR bacteria in Cambodia. (A) The prototype used in this study and (B) current commercial type are shown.

Supplementary Figure 2 | Multiple sequence alignment of carbapenemase genes analyzed by MAFFT v7.475. (A) Comparison between the bla NDM $-5^{\text {-like }}$ gene in E. coli pNIPH17_0020_1 (from on-site ONT analysis) and the reference gene (bla $\mathrm{NDM}_{-5}$ in accession no. JN104597), (B) comparison between the blaoxA-181-like gene in E. coli PNIPH17_0036_1 (from on-site ONT analysis) and the reference sequence (blaoxA-181 in accession no. CM004561), and (C) comparison between the blaoxA-23-like sequence in A. baumannii NIPH17_0019 (from on-site ONT analysis) and the reference sequence (blaOXA-23 in accession no. AY795964) are shown.

Supplementary Figure 3 | Linear comparison of AbaR4-containing genomic regions in A. baumannii NIPH17_0019 harboring two copies of blaOXA-23. Two sets of AbaR4 with blaoxA-23 in A. baumannii NIPH17_0019: (A) 216,633233,441 nt region inserted within comM and (B) 3,924,674-3,941,483 nt region inserted within mutY in accession no. AP024415, and (A,B) comparison of both the uncorrected sequences (from on-site ONT analysis) and corrected sequences (from subsequent ONT + Illumina analysis) are shown. Red, blue, black, and gray arrows indicate carbapenemase gene (b/aOXA-23), mobile gene elements (MGE), pseudogenes disrupted by AbaR4 insertion (Pseudo), and other genes (Others), respectively. The colors in comparison of sequences show percent identity.

Supplementary Table 1 | Summary of whole-genome sequencing and bioinformatics analysis of AMR bacteria isolated in Cambodia in this study. Bacterial isolates, nucleotides of raw reads of MinION sequencing and the corrected reads, genome size of the uncorrected sequence (from on-site ONT analysis) and corrected sequence (from subsequent ONT + Illumina analysis) are shown. Also, AMR genes (ARG) associated with resistance to $\beta$-lactams, aminoglycosides (AG), and fluoroquinolones (FQ), virulence genes, and accession nos. of sequences are shown.

Chmelnitsky, I., Navon-Venezia, S., Strahilevitz, J., and Carmeli, Y. (2008) Plasmid-mediated qnrB2 and carbapenemase gene bla(KPC-2) carried on the same plasmid in carbapenem-resistant ciprofloxacin-susceptible Enterobacter cloacae isolates. Antimicrob. Agents Chemother. 52, 2962-2965. doi: 10.1128/ aac.01341-07

Conlan, S., Thomas, P. J., Deming, C., Park, M., Lau, A. F., Dekker, J. P., et al. (2014). Single-molecule sequencing to track plasmid diversity of hospitalassociated carbapenemase-producing Enterobacteriaceae. Sci. Transl. Med. 6:254ra126. doi: 10.1126/scitranslmed.3009845

Corvec, S., Poirel, L., Naas, T., Drugeon, H., and Nordmann, P. (2007). Genetics and expression of the carbapenem-hydrolyzing oxacillinase gene blaOXA-23 in Acinetobacter baumannii. Antimicrob. Agents Chemother. 51, 1530-1533. doi: 10.1128/aac.01132-06

Faria, N. R., Quick, J., Claro, I. M., Theze, J., de Jesus, J. G., Giovanetti, M., et al. (2017). Establishment and cryptic transmission of Zika virus in Brazil and the Americas. Nature 546, 406-410.

Gandra, S., Alvarez-Uria, G., Turner, P., Joshi, J., Limmathurotsakul, D., and van Doorn, H. R. (2020). Antimicrobial resistance surveillance in low- and 
middle-income countries: progress and challenges in eight South Asian and Southeast Asian Countries. Clin. Microbiol. Rev. 33:e00048-19.

Hamidian, M., and Hall, R. M. (2011). AbaR4 replaces AbaR3 in a carbapenemresistant Acinetobacter baumannii isolate belonging to global clone 1 from an Australian hospital. J. Antimicrob. Chemother. 66, 2484-2491. doi: 10.1093/jac/ dkr356

Hamidian, M., Hawkey, J., Holt, K. E., and Hall, R. M. (2015). Genome sequence of Acinetobacter baumannii strain D36, an antibiotic-resistant isolate from lineage 2 of global clone 1. Genome Announc. 3:e01478-15.

Hamidian, M., and Nigro, S. J. (2019). Emergence, molecular mechanisms and global spread of carbapenem-resistant Acinetobacter baumannii. Microb. Genom. 5:e000306.

Hattori, T., Kawamura, K., and Arakawa, Y. (2013). Comparison of test methods for detecting metallo-beta-lactamase-producing Gram-negative bacteria. Jpn. J. Infect. Dis. 66, 512-518. doi: 10.7883/yoken.66.512

Jain, M., Olsen, H. E., Paten, B., and Akeson, M. (2016). The Oxford Nanopore MinION: delivery of nanopore sequencing to the genomics community. Genome Biol. 17:239.

Li, H. (2016). Minimap and miniasm: fast mapping and de novo assembly for noisy long sequences. Bioinformatics 32, 2103-2110. doi: 10.1093/bioinformatics/ btw152

Liu, Y., Feng, Y., Wu, W., Xie, Y., Wang, X., Zhang, X., et al. (2015). First report of OXA-181-producing Escherichia coli in China and characterization of the isolate using whole-genome sequencing. Antimicrob. Agents Chemother. 59, 5022-5025. doi: 10.1128/aac.00442-15

Malchione, M. D., Torres, L. M., Hartley, D. M., Koch, M., and Goodman, J. L. (2019). Carbapenem and colistin resistance in Enterobacteriaceae in Southeast Asia: review and mapping of emerging and overlapping challenges. Int. J. Antimicrob. Agents 54, 381-399. doi: 10.1016/j.ijantimicag.2019. 07.019

Nadimpalli, M. L., Lauzanne, A., Phe, T., Borand, L., Jacobs, J., Fabre, L., et al. (2019). Escherichia coli ST410 among humans and the environment in Southeast Asia. Int. J. Antimicrob. Agents 54, 228-232. doi: 10.1016/j. ijantimicag.2019.05.024

Nigro, S. J., and Hall, R. M. (2016). Structure and context of Acinetobacter transposons carrying the oxa23 carbapenemase gene. J. Antimicrob. Chemother. 71, 1135-1147.

Poirel, L., Dortet, L., Bernabeu, S., and Nordmann, P. (2011a). Genetic features of blaNDM-1-positive Enterobacteriaceae. Antimicrob. Agents Chemother. 55, 5403-5407. doi: 10.1128/aac.00585-11

Poirel, L., Walsh, T. R., Cuvillier, V., and Nordmann, P. (2011b). Multiplex PCR for detection of acquired carbapenemase genes. Diagn. Microbiol. Infect. Dis. 70, 119-123. doi: 10.1016/j.diagmicrobio.2010.12.002

Quick, J., Loman, N. J., Duraffour, S., Simpson, J. T., Severi, E., Cowley, L., et al. (2016). Real-time, portable genome sequencing for Ebola surveillance. Nature 530, 228-232.

Santajit, S., and Indrawattana, N. (2016). Mechanisms of Antimicrobial Resistance in ESKAPE Pathogens. Biomed. Res. Int. 2016:2475067.

Seputiene, V., Povilonis, J., and Suziedeliene, E. (2012). Novel variants of AbaR resistance islands with a common backbone in Acinetobacter baumannii isolates of European clone II. Antimicrob. Agents Chemother. 56, 1969-1973. doi: 10.1128/aac.05678-11

Sugawara, Y., Akeda, Y., Sakamoto, N., Takeuchi, D., Motooka, D., Nakamura, S., et al. (2017). Genetic characterization of blaNDM-harboring plasmids in carbapenem-resistant Escherichia coli from Myanmar. PLoS One 12:e0184720. doi: 10.1371 /journal.pone. 0184720
Suwantarat, N., and Carroll, K. C. (2016). Epidemiology and molecular characterization of multidrug-resistant gram-negative bacteria in Southeast Asia. Antimicrob. Resist. Infect. Control 5:15.

Tada, T., Miyoshi-Akiyama, T., Shimada, K., Nga, T. T., Thu le, T. A., Son, N. T., et al. (2015). Dissemination of clonal complex 2 Acinetobacter baumannii strains co-producing carbapenemases and 16S rRNA methylase ArmA in Vietnam. BMC Infect. Dis. 15:433. doi: 10.1186/s12879-015-1171-x

Tornimbene, B., Eremin, S., Escher, M., Griskeviciene, J., Manglani, S., PessoaSilva, C. L., et al. (2018). Resistance surveillance system early implementation 2016-17. Lancet Infect. Dis. 18, 241-242. doi: 10.1016/s1473-3099(18)30 060-4

Turton, J. F., Ward, M. E., Woodford, N., Kaufmann, M. E., Pike, R., Livermore, D. M., et al. (2006). The role of ISAbal in expression of OXA carbapenemase genes in Acinetobacter baumannii. FEMS Microbiol. Lett. 258, 72-77. doi: 10.1111/j.1574-6968.2006.00195.x

Tzouvelekis, L. S., Markogiannakis, A., Psichogiou, M., Tassios, P. T., and Daikos, G. L. (2012). Carbapenemases in Klebsiella pneumoniae and other Enterobacteriaceae: an evolving crisis of global dimensions. Clin. Microbiol. Rev. 25, 682-707. doi: 10.1128/cmr.05035-11

WHO (2015). Global Action Plan on Antimicrobial Resistance. Available online at: https://apps.who.int/iris/bitstream/handle/10665/193736/9789241509763_ eng.pdf? sequence $=1$ \&isAllowed $=y$

WHO (2019). Cambodia Laboratory Information System (CamLIS): A National Web-Based Laboratory Information System to Enhance Public Health in Cambodia. Available online at: https://www.who.int/cambodia/news/detail/ 11-02-2019-cambodia-laboratory-information-system-(camlis)-a-nationalweb-based-laboratory-information-system-to-enhance-public-health-incambodia

Woodford, N., Ellington, M. J., Coelho, J. M., Turton, J. F., Ward, M. E., Brown, S., et al. (2006). Multiplex PCR for genes encoding prevalent OXA carbapenemases in Acinetobacter spp. Int. J. Antimicrob. Agents 27, 351-353. doi: 10.1016/j. ijantimicag.2006.01.004

Wu, J. J., Ko, W. C., Tsai, S. H., and Yan, J. J. (2007). Prevalence of plasmidmediated quinolone resistance determinants QnrA, QnrB, and QnrS among clinical isolates of Enterobacter cloacae in a Taiwanese hospital. Antimicrob. Agents Chemother. 51, 1223-1227. doi: 10.1128/aac.01195-06

Zurfluh, K., Poirel, L., Nordmann, P., Klumpp, J., and Stephan, R. (2015). First detection of Klebsiella variicola producing OXA-181 carbapenemase in fresh vegetable imported from Asia to Switzerland. Antimicrob. Resist. Infect. Control 4:38.

Conflict of Interest: HY was employed by the company MicroSKY Lab., Inc., Tokyo, Japan. PB and BW were employed by the company Bento Bioworks Ltd., London, United Kingdom.

The remaining authors declare that the research was conducted in the absence of any commercial or financial relationships that could be construed as a potential conflict of interest.

Copyright (C) 2021 Hirabayashi, Yanagisawa, Takahashi, Yahara, Boeing, Wolfenden, Nov, Lorn, Veng, Ann, Darapheak, Shibayama and Suzuki. This is an open-access article distributed under the terms of the Creative Commons Attribution License (CC BY). The use, distribution or reproduction in other forums is permitted, provided the original author(s) and the copyright owner(s) are credited and that the original publication in this journal is cited, in accordance with accepted academic practice. No use, distribution or reproduction is permitted which does not comply with these terms. 